\title{
Understanding Treasures: The Application of Micro-Computed Tomography on the Study of the Blaschka Models
}

\author{
Efstratia Verveniotou ${ }^{1}$, Dan Sykes ${ }^{2}$, Helen Walker $^{3}$, Chris Collins ${ }^{1}$, Farah Ahmed $^{2}$ \\ 1. Science Facilities, Conservation Unit, The Natural History Museum, London, United Kingdom \\ 2. Science Facilities, Imaging and Analysis Centre, The Natural History Museum, London, United \\ Kingdom \\ 3. Programme and Production, Design and Conservation, The Natural History Museum, London, United \\ Kingdom
}

Admired for their intricate details, prized for their aesthetic value and cherished as a window to the scientific world of the Victorians, the work of German glass artisans Leopold and Rudolph Blaschka has been under continuous study during the last few years [1]. That could not be more true for the 187 Blaschka marine invertebrate models of sea anemones, nudibranchs, cephalopods, jellyfish, protozoans and corals that the Natural History Museum currently holds. This paper examines the use of MicroComputed Tomography (Micro-CT) in shaping the conservation strategy of these intricately made models and presents how this technique offered an invaluable means to provide better care for the collection.

Each model is delicately and intricately made by fusing or adhering glass while employing a range of organic materials and often-fine metal wires to support the structures. The inherent unstable nature of the glass and that of the coatings and adhesives originally used along with years of pollution and inappropriate storage that the models have been exposed to left the models extremely fragile and prone to fractures. For the past ten years conservators at the NHM have carried out bespoke conservation treatments based on analysis using a range of techniques including Fourier-Transform Infrared microscopy, Raman spectroscopy, Scanning Electron Microscopy Ion Chromatography and X-ray Diffraction [2].

The analytical techniques used in the past although suit the conservation of individual specimens, they do not always enable quick examination of specimens, or analysis of models that cannot have samples taken from. Given the large number of collections, Micro-CT has proven a useful alternative in quickly and routinely identifying or distinguishing different types of materials used, when studied next to data from previous analyses such as SEM- EDX. This is particularly evident in the distinction between the different types of glass used. For example, the use of lead glass can be easily mapped and its use better seen as the predominant material for fine detail, as seen in Figure 1.

Micro-CT has also offered the opportunity to examine each component of the model and its correlation to the general structure. This allows the model to be virtually dissected and viewed under any possible angle, offering a unique opportunity to identify the location of detached components. This is particular important with models like the radiolarians and the jellyfish as some of their features are repetitive and the points where they need to be attached are not visually accessible or clear. Views of the internal structure of the model has also allowed the conservators to better understand the manufacturing techniques and help address issues with the potential stability of various features.

Micro-CT data has also been used to develop supports for the models in storage and display. Production 
of a mount from the digital data overcomes the difficulties that come with handling fragile specimen for traditional mount making and therefore, minimizing the risk while enabling a closer fit to the profile of the specimen. Using rapid prototyping the data has been turned into bespoke mounts that have then been cast in more stable materials, as seen in Figure 2. Those mounts are of paramount importance to the safe storage and display of the specimens as they allow specimens to be rotated on display while addressing the risks of handling them directly.

The rendered data from Micro-CT also allow a more detailed level of condition reporting. Each feature can be isolated measured and counted for giving the opportunity to the conservator to check on its condition at any given time. That is particularly important for the models that rely on fine metal wires to support various features, as they can be accidentally bent or moved as adhesives fail. In a museum environment Micro-CT has offered a new point of engagement with the museums exhibition design teams and public as it offers a contemporary, dramatic and yet scientific way to see an art form.

\section{References:}

[1] Miller C G \& Lowe M, The Natural History Museum Blaschka Collections, Historical Biology Volume 20 (2008) p. 51-62

[2] Brierley L. Art Forms in Nature: Examination and Conservation of a Blaschka Glass Model of the Protozoan Aulosphaera elegantissima, Studies in Conservation (2009) p. 255-267

[3] Authors would like to thank Meg Macdonald and RapidformRCA for their assistance with the production of the bespoke mount and curators Dr. Giles Miller and Miranda Lowe for their support.

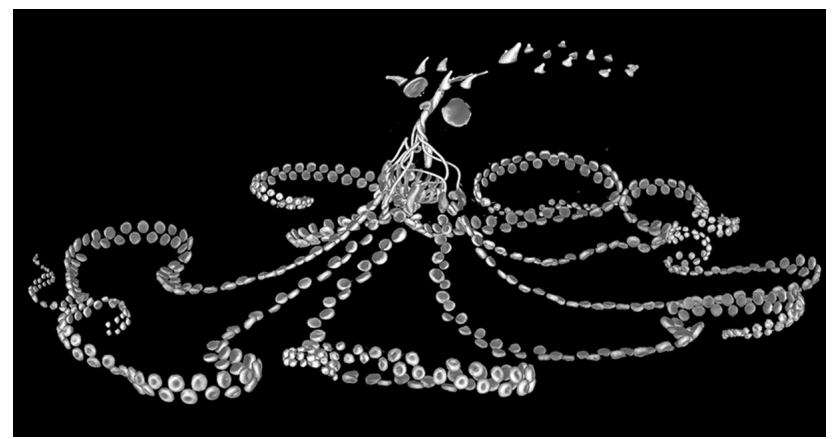

Figure 1. Octopus vulgaris, CT- scan data separating the lead rich glass from the structure

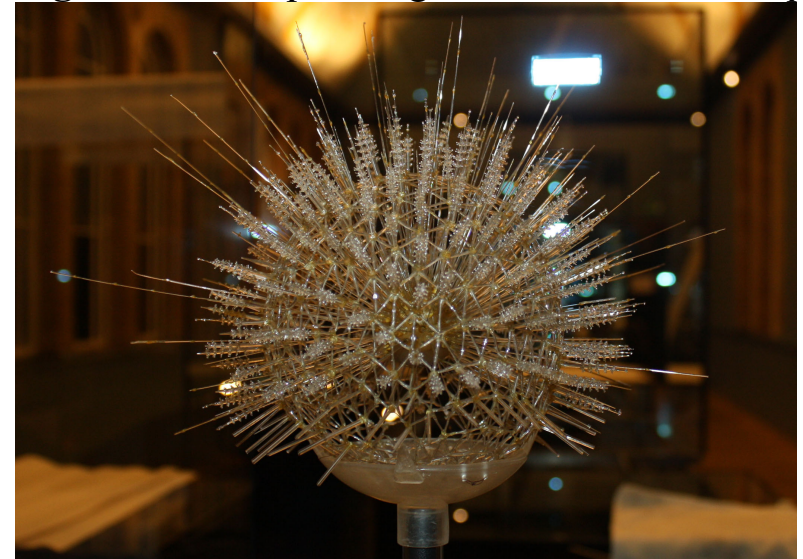

Figure 2. Aulosphaera elegantisssima radiolarian, on rapid prototyped mount 